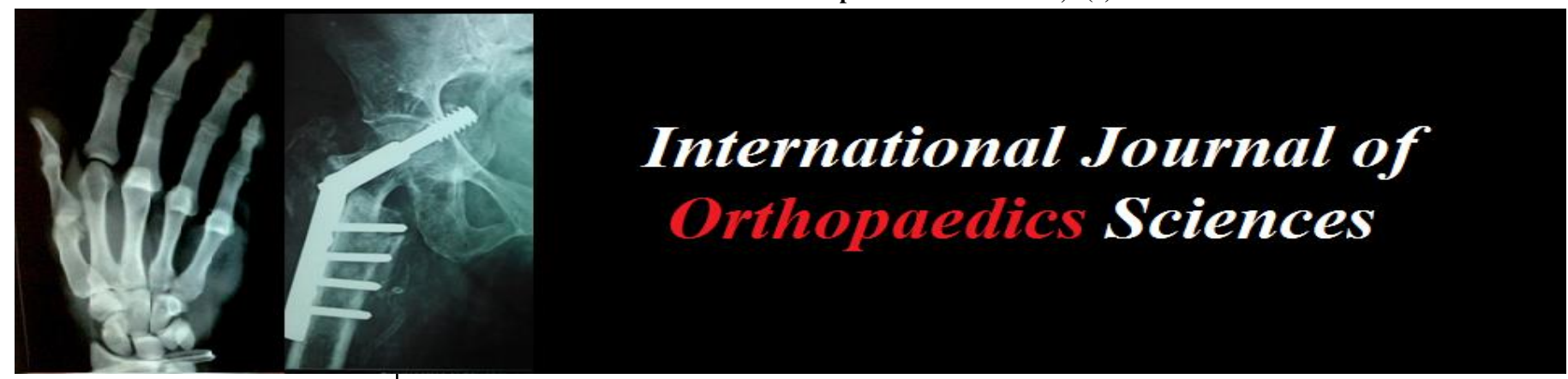

E-ISSN: 2395-1958

P-ISSN: 2706-6630

IJOS 2019; 5(4): 878-881

(C) 2019 IJOS

www.orthopaper.com

Received: 21-08-2019

Accepted: 25-09-2019

Dr. Dhanraj Meena

Resident, Govt. Medical College

Kota, Rajasthan, India

Dr. Ashok Kumar Tiwari

Senior Professor, Govt. Medical

College Kota, Rajasthan, India

Corresponding Author:

Dr. Ashok Kumar Tiwari

Senior Professor, Govt. Medical

College Kota, Rajasthan, India

\section{Results of management of tibia shaft fractures in children with titanium elastic nailing}

\author{
Dr. Dhanraj Meena and Dr. Ashok Kumar Tiwari
}

DOI: https://doi.org/10.22271/ortho.2019.v5.i4o.1789

\section{Abstract}

Background: Closed reduction and casting is effective form of treatment of tibial shaft fractures in children. Elastic nailing is of tibia fracture in children has gained popularity. Elastic nailing allows early discharge from hospital and early mobilization, does not usually require casting, and avoids repeated remanipulation often needed in non-operative treatment to maintain axial alignment. The purpose of this study was to assess the outcome of unstable tibial shaft fractures fixation with titanium elastic intramedullary nails.

Material and Methods: In this hospital based prospective study, 32 patients with tibial shaft fractures were treated with titanium elastic nails in the Department of Orthopaedic, Govt. Medical College and Associated group of hospitals, Kota during the September 2017- December 2019. Post operatively outcomes were classified as excellent, satisfactory or poor according to Flynn's criteria for flexible nail fixation.

Result: There were 6(19\%) girls and $26(81 \%)$ boys in the present study. RTA was the most common mode of injury accounting for $26(81.25 \%$ ) cases, Average duration between trauma and surgery was 2.7 days in our study, the mean time for union found to be 12.4 weeks. Superficial infection was seen in only $1(3.1 \%)$ case. Two patient complains nail end irritation. In the present study, the final outcome was excellent in $26(81.25 \%)$ cases, satisfactory in $4(12.5)$ cases and there $6.25 \%$ poor outcome cases.

Conclusion: Tens nails is method of choice for tibia shaft fracture in pediatric patients, because it allow early mobilization, short hospital stays, and higher parent satisfaction.

Keywords: Tibia fractures, elastc nailing, children, tens nail

\section{Introduction}

Pediatric tibial fractures are the third most common pediatric long bone fracture after the femur and forearm ${ }^{[1]}$. They representing $15 \%$ of all pediatric fractures ${ }^{[2]}$. The most common location of tibia fractures is in the distal third of the tibia (50-70\%) followed by middle and then proximal (less common) thirds. Management of pediatric tibial shaft fracture is individualized based on age, co-existing injuries, and associated soft tissue and neurovascular injury. Closed reduction and GT pop cast is conservative treatment of tibial shaft fractures in children. This is particularly the case for undisplaced or minimal displaced tibial shaft fractures. There is possibility of loss of reduction, shortening, angulations and malunion after closed reduction and casting. Plaster immobilization has its own complication like pressure sores, nerve palsies, soiling of skin and plaster, breakage of plaster, joint stiffness. The child is immobilized and needs an attendant for personal care. Conservative treatment give good results in children under 6 years of age.

Over the fast few decades management of pediatric tibial fractures has shifted more towards operative interventions because of quick recovery, shorter rehabilitation period, less immobilization, lack of stiffness of adjoining joints and less psychological impact to the children ${ }^{[3]}$. External fixation, open reduction and internal fixation with plate and screw fixation, percutanious $\mathrm{k}$-wires, flexible intramedullary nailing or stainless steel nails are other treatment options for tibial shaft fractures in children.

Elastic nailing (TENS) of tibia fracture in children has gained popularity because of its high effectiveness and less complication rate ${ }^{[4]}$. Elastic nail are load sharing device act as internal splint, allow early mobilization and early discharge from hospital ${ }^{[5]}$. Elatstic usually does not require casting and avoid repeated re- manipulations often needed in nonoperative treatment to 
maintain axial alignment ${ }^{[6]}$. The advantage of elastic intramedullary nails include closed insertion, small incision during surgery, minimum soft tissue injury, preservation of fracture hematoma, low infection rate ${ }^{[7]}$, and the most importantly, a physeal sparing entery point ${ }^{[8]}$. Elastic nailing has high union rates, effective and safe treatment for closed and open tibia fracture in children ${ }^{[9]}$.

The aim of present study was to assess the results of management of tibial shaft fractures in children with titanium elastic intramedullary nails.

\section{Materials and Methods}

This hospital based prospective study was conducted in the Department of Orthopaedic, Govt. Medical College and Associated group of hospitals, Kota during the September 2017- December 2019. 35 patients were included in present study out of which 3 patients lost to final follow up. Final assessment of results done on 32 patients.

\section{Inclusion criteria}

1. Age 6 to 14 years both male and females

2. Unstable unilateral tibia diaphyseal fractures.

3. Grade 1 and 2 compound fracture.

4. Failed closed reduction with unstable alignment.

\section{Exclusion criteria}

1. Patients with malunited, non-union tibial fractures.

2. Patients who are medically unstable, at extremely poor anesthesia risk.

3. Open fractures Gustilo-Anderson type 3 (c) fractures.

4. Patients with same side femur fractures.

5. Bilateral tibia fractures.

6. Patients with age < 6yrs and $>14$ years.

7. Metaphyseal fractures.

\section{Operative procedure}

The patients were taken up for surgery under spinal or general anesthesia. Place the patient supine on radiolucent table and bump is placed below the knee .tourniquet is applied to upper thigh. After adequately Draping and painting with use of fluoroscopy, the starting point for nail entery hole is 1.5 to 2.0 $\mathrm{cm}$ distal to the physis. Place the prebent nail on an inserter and insrt it from the side opposite the tibial displacement in antegrade fashion. Under the fluoroscopic guidance, slide the nails along the opposite cortex until fracture is reached. Reduce the fracture and advance the nail across the fracture .embed the nail in distal tibial metaphysic without violating the cortex or the physis. Place the second nail from the other side in a similar fashion.
Final outcome after 6 month is graded as excellent, satisfactory, or poor based on criteria described by Flynn et al.

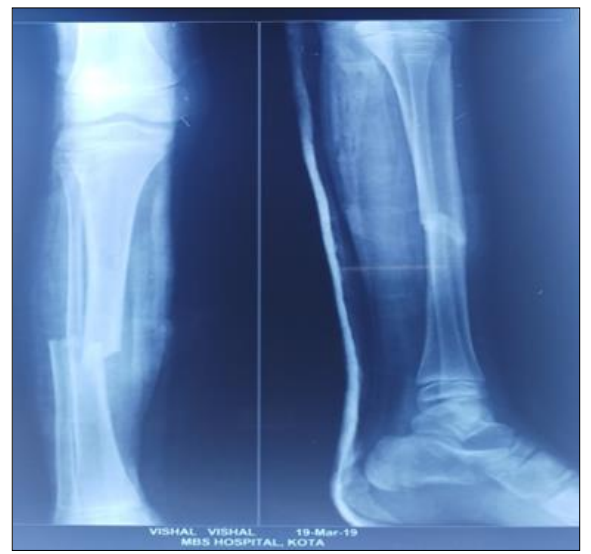

Fig 1: Preoperative X-ray

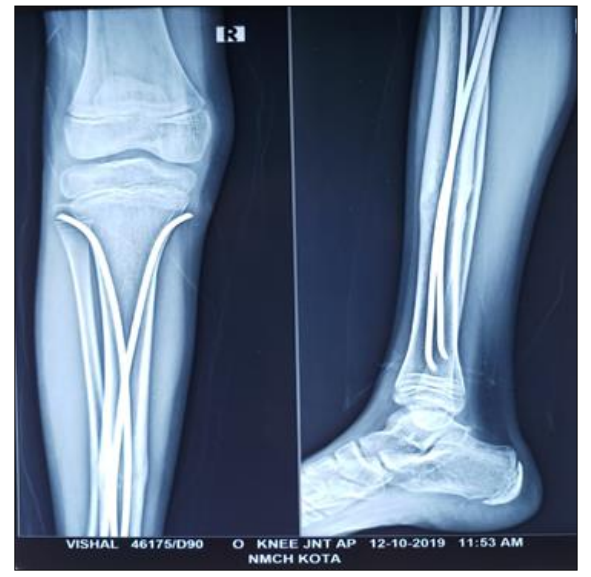

Fig 2: Follow up at 6 month

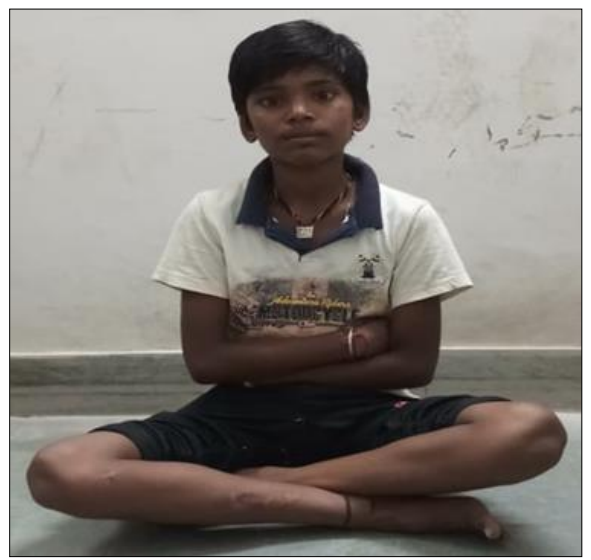

Fig 3: Sitting cross leg

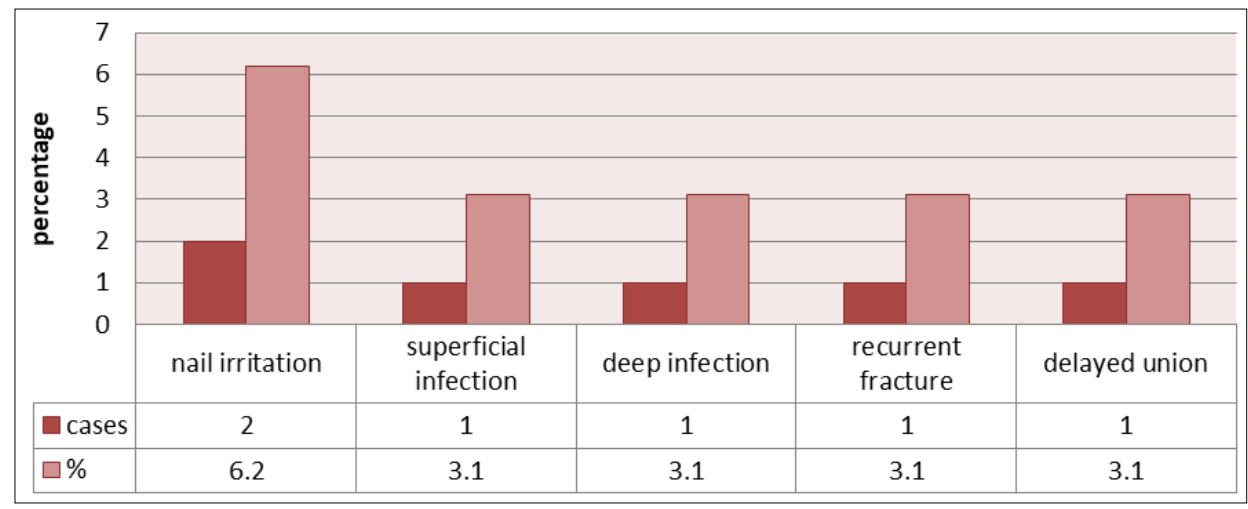

Graph 1: Complications 


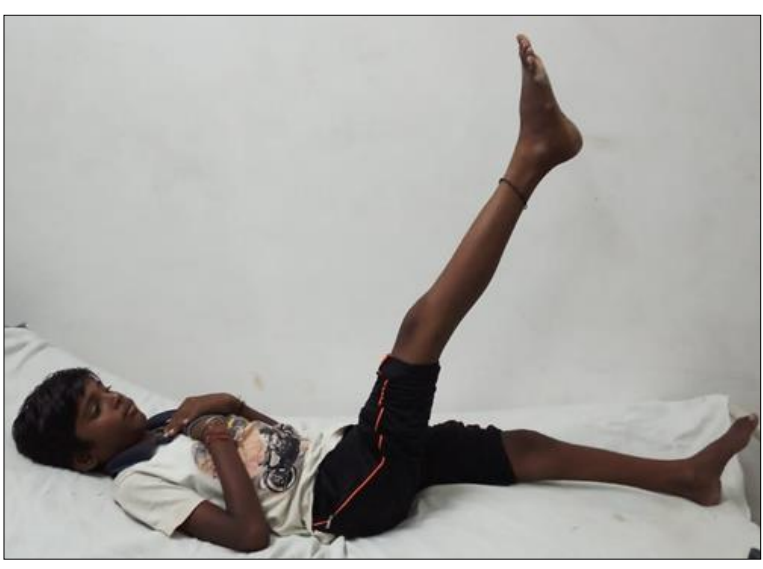

Fig 4: Knee Extension

\section{Results}

In present study, $18.75 \%$ of patients were age 6 to 8 years and $81.25 \%$ of patients were between 9 to 14 years of age. In present study, $19 \%$ of patients were female and $81 \%$ of patients were male. Majority $(81.25 \%)$ of patients had trauma due to road traffic accident and $15.62 \%$ due to self-fall and 1 case $(3.13 \%)$ due to sport related injury. In our study, right side affected more than left side. In present study, transverse fractures cases were $53.13 \%$, short oblique cases was 43.75 $\%$ and 1 case was communited fracture. Fractures involving the middle shaft was $62.5 \%$, distal third-37.5 \% and there was no case of proximal one third of fracture of tibia.

In present study 29 cases $(90.7 \%)$ in our series operated within 4 days. Average duration of surgery was 2.7 days in our study. In our study average duration of surgery was 48.9 minutes. In majority of patients $71.9 \%$ partial weight bearing started at 6 weeks. The average time of partial weight bearing was 6.4 weeks. The average duration of hospital stay in our study was 6.1 days.

Most of cases (75\%) in our study radiologically and clinically (no pain and tenderness at fracture site) united up 12 weeks of surgery.one case delay union was noted. Superficial infection was seen in only 1 case $(3.1 \%)$ in our study. Late deep infection or osteomylitis seen in 1 case. Refracture was present in 1 case at the time of final follow up due to self-fall. Nail irritation was present in 2 cases in our study.

In our study, the final outcome was excellent in $81.25 \%$, satisfactory or good in $12.5 \%$ cases and poor outcome was reported in $(6.25 \%)$ only.

\section{Discussion}

The traditional method of managing pediatric tibial fracture conservatively given good functional results. The difficulties of non -surgical treatment while keeping children in plaster cast needs an alternative approach. Working parents, nuclear family with challenging home care, academics leaves and patient discomfort need to be considered while opting for non-surgical treatment.

The ideal implant to treat pediatric tibial fracture, should be load sharing it should maintain alignment, allow early mobilization, and should not cross the physis. Since 1995, titanium elastic intramedullay nails to stabilize pediatric long bone fractures in use where surgery is indicated.

Wudbhav N. Sankar et al. in their study allowed full weight bearing between $5.7-11.6$ weeks an average of 8.65 weeks. The average of full weight bearing for all tibia fracture in children was $>20$ weeks in study of Shrivastava et al, (2008). J.M. Flynn et al. reported $4(1.7 \%)$ cases, Griffet J et al reported 4 patients $(5 \%)$ suffered from superficial infection. In Gordon et al (2007) series, one patients with Gustilo grade 2 open fracture was diagnosed with osteomylitis at fracture site after the bony union. Wudbhav N. Sankar in their study of 19 tibial shaft fractures reported no leg length discrepancy which is similar to our results. Valamastha et al (2006) reported limb length inequality of $<15 \mathrm{~mm}$ in $3.6 \%$ of patients which is contrary to our study result. The varus and valgus malalignment not occurred in our study Heinrich SD, et al (1994) reported $5^{\circ}$ of varus angulation in one child in their study and $11 \%$ of fractures had an average varus or valgus malalignment of $6^{\circ}$. In the present study, no patients had anteroposterior angulation. Ozturkman Y, et al noted an anterior angulation of $7^{\circ}$ and a posterior angulation of $6^{\circ}$ in 2 patients respectively. In the present series, nail back out was not seen in any of the cases. Carrey T.P. et al out of 38 cases, noted nail back out in one case in their study, which necessitated early removal.

Irritation at nail entry site was present in 2 cases out of 32 in our study. In series of Wudbhav N. Sankar et al (2007) irritation was present in five patients $(26 \%)$.

In the present study, the final outcome was excellent in 26 $(81.25 \%)$ cases, satisfactory in $4(12.5 \%)$ cases and there $6.25 \%$ poor outcome cases (2 cases). Wudbhav N. Sankar (2007) in their study of 19 tibial shaft fractures reported 12 $(63.15 \%)$ excellent, $6(31.57 \%)$ satisfactory and $1(5.26 \%)$ poor result. Gamal El Adl et al. (2009) in their study of 66 children with 48 femoral and 25 tibial shaft fractures reported $(75.8 \%)$ excellent, $24.2 \%$ satisfactory and no poor results ${ }^{[2]}$.

\section{Conclusion}

The trational method of managing pediatric tibial fractures conservatively given good results. The most predictable method to maintain the reduction of displaced tibial fractures including length and roation is titanium elastic intramedullary nail fixation. Titanium elastic nailing seems to be more physiological and effective method of treatment of tibial shaft fractures in 6-14 years old children. It is simple, rapid and safe procedure with advantages of early union, early mobilization and early return to function with minimum complications. Elastic medullary nailing advantages make it a valuable choice to consider in managing children shaft tibial fractures.

\section{References}

1. Shannak AO. Tibial fractures in children: Followup study. J Pediatr Orthop. 1988; 8:306-310.

2. Hansen BA, Greiff S, Bergmann F. Fractures of the tibia in children .Acta Orthop Scand. 1976; 47:448-453.

3. Wudbhav N. Sankar, Kristofer J. Jones, B. David Horn, and Lawrence Wells. Titanium elastic nails for pediatric tibial shaft fractures. J Child Orthop 2007 November; 1(5):281-286.

4. Flynn JM, Hresko T, Reynolds RA. Titanium elastic nails for paediatric femur fractures: A multi-center study of early results with analysis of complications. J Pediatr Orthop. 2001; 21:4-8.

5. Sauli A Palmu, Sampo Auro, Martina Lohman, Reijo T Paukku, Jari I Peltonen, Yrjänä Nietosvaara. Tibial fractures in children, Acta Orthopaedica. 2014; 85:5:513517.

6. Carey TP, Galpin RD. Flexible intramedullary nail fixation of pediatric femoral fractures. Clin Orthop. 1996; $332: 110-118$ 
7. Metaizeau J. Stable elastic intramedulary nailing of fractures of the femur in children. J Bone Joint Surg Br. 2004; 86:954-957.

8. Ahmed Labib Zarada .Flexible intramedullary nails for unstable fractures of the tibia in children: A retrospective evaluation of effectiveness, Egypt Orthop J. 2014; 49:281-285

9. Marengo L, et al. Elastic stable intramedullary nailing (ESIN) in paediatric femur and tibia shaft: comparasion between titanium and stainless steel nails Injury, 2018. 